\title{
Computational analysis of the LRRK2 interactome
}

Claudia Manzoni, Paul Denny, Ruth C Lovering, Patrick A Lewis

LRRK2 was identified in 2004 as the causative protein product of the Parkinson's disease locus designated PARK8. In the decade since then, genetic studies have revealed at least 6 dominant mutations in LRRK2 linked to Parkinson's disease, alongside one associated with cancer. It is now well established that coding changes in LRRK2 are one of the most common causes of Parkinson's. Genome wide association studies (GWAs) have, more recently, reported single nucleotide polymorphisms (SNPS) around the LRRK2 locus to be associated with risk of developing sporadic Parkinson's disease and inflammatory bowel disorder. The functional research that has followed these genetic breakthroughs has generated an extensive literature regarding LRRK2 pathophysiology; however there is still no consensus as to the biological function of LRRK2. To provide insight into the aspects of cell biology that are consistently related to LRRK2 activity, we analysed the plethora of candidate LRRK2 interactors available through the BioGRID and IntAct data repositories. We then performed GO terms enrichment for the LRRK2 interactome. We found that, in two different enrichment portals, the LRRK2 interactome was associated with terms referring to transport, cellular organization, vesicles and the cytoskeleton. We also verified that 21 of the LRRK2 interactors are genetically linked to risk for Parkinson's disease or inflammatory bowel disorder. The implications of these findings are discussed, with particular regard to potential novel areas of investigation. 


\section{Computational analysis of the LRRK2 interactome} Claudia Manzoni ${ }^{1,2 *}$, Paul Denny ${ }^{3}$, Ruth C. Lovering ${ }^{3}$ and Patrick A. Lewis ${ }^{1,2,4}$

1. School of Pharmacy, University of Reading, Whiteknights, Reading, RG6 6AP, UK; 2. UCL Institute of Neurology, Queen Square, London, WC1N 3BG, UK; 3. UCL Institute of Cardiovascular Science, London, WC1E 6BT, UK. 4. Centre for Integrated Neuroscience and Neurodynamics, University of Reading, Whiteknights, Reading, RG6 6AP, UK

*To whom correspondence should be addressed: c.manzoni@reading.ac.uk

\section{Introduction}

LRRK2 is the most frequently mutated gene in familial Parkinson's disease (PD) (Paisán-Ruíz et al., 2004; Zimprich et al., 2004), and has also been identified as a risk locus for the sporadic form of the disease (Nalls et al., 2014). There are additional reports implicating allelic variants at the $L R R K 2$ locus with increased risk of developing inflammatory bowel disorder (IBD) (Jostins et al., 2012). LRRK2 has also been linked to susceptibility to multibacillary Leprosy (Zhang et al., 2009), although reproducibility of the data is controversial (Marcinek et al., 2013; Zhang et al., 2011). Furthermore, genetic, as well as epidemiological data, supports a role for $L R R K 2$ in cancer for certain populations (Ruiz-Martínez et al., 2014; Inzelberg et al., 2012; Saunders-Pullman et al., 2010). The protein product of the LRRK2 gene, Leucine Rich Repeat Kinase 2 (LRRK2) is a large enzyme hosting kinase and GTPase functions surrounded by protein-protein interactions domains (Greggio et al., 2008). It is now well recognized that there are complex regulatory events linking the two enzymatic activities within LRRK2. These include, although are likely not to be limited to, regulation of dimerization by GTP and/or GDP binding and auto-phosphorylation events (Taymans et al., 2011). The importance of LRRK2 in a number of human diseases has motivated many different groups to try and solve the conundrum of its pathophysiological activity. This has led to a large and increasing body of information emerging from functional studies of LRRK2. Despite the bulk of existing data, there is no consensus on the phosphorylation targets of the LRRK2 kinase domain, or with regard to the binding partners with which LRRK2 interacts. Many different functions have been reported to be associated with LRRK2, ranging from cytoskeleton organization to vesicle trafficking, from synaptic activities to autophagy, from mitochondria homeostasis to protein synthesis, involving multiple different signalling 
33 cascades, such as the m-TOR and Wnt pathways (Paisán-Ruiz et al., 2013). None of these have been 34 categorically proven; the only reproducible data at the moment appears to be the interaction between 35 LRRK2 and 14-3-3 that has been shown to regulate LRRK2 localization in the cell (Dzamko et al., 36 2010). The reasons for this volume of different and sometimes contradictory, literature may be the

\section{Materials and Methods}

\section{LRRK2 interactors: IntAct database}

61 http://www.ebi.ac.uk/intact/

62 The IntAct database (Kerrien et al., 2006; Hermjakob et al., 2004) was queried for LRRK2 (human, 63 Q5S007). The list of interactors was filtered to remove the spoke expanded co-complex (i.e. 
annotations derived by an expansion algorithm sampling co-complexes data into binary interactions and therefore listing proteins which may not interact directly with LRRK2). This reduces the number of proteins not directly interacting with LRRK2, but does not remove all of these proteins. The MITAB 2.7 file was downloaded (26 th July 2014) and imported into an Excel spreadsheet. Filter \#1 screened the taxid, to retain only human-human interactions (annotation removed for non-human taxid or chemicals). Filter \#2 was applied to remove multiple entries (in the same publication) when: i) Interactor A was used as bait in the first experiment and as prey in the second one and interactor B vice versa; ii) the same experiment was performed using differently tagged A and B interactors (e.g. GST Vs FLAG Vs HA). To apply filter \#2 the following cut-off was established: 2 entries with the same "Publication Identifier(s)" and "\#ID(s) interactor A and ID(s) interactor B", were considered duplicated (i.e. one of them to be removed) if "Interaction detection method(s)" AND "Host organism(s)" were identical.

As a general note, protein fragments can be retrieved with the same UniProtKB identifier as for the entire protein (e.g. MAP1B catalytic domain (P46821-PRO_0000018605) has the same identifier as the entire MAP1B protein (P46821)); protein isoforms are queried by the same UniProtKB identifier as for the principal isoform (e.g. DVL2 isoform 2 (O14641-2) has the same identifier as DVL2 (O14641)).

For this reason no discrimination was made between protein fragments and the entire protein; nor between different protein isoforms and the principal isoform.

\section{LRRK2 interactors: BioGrid database}

http://thebiogrid.org/

BioGrid database (Chatr-Aryamontri et al., 2012; Breitkreutz, Stark \& Tyers, 2003) was queried for LRRK2 (human, Q5S007). The list of interactions was downloaded (12 ${ }^{\text {th }}$ August 2014) as a TAB 2.0

file and imported into an Excel spreadsheet to be analysed. Filters \#1 and \#2 were applied, as described above for the processing of the IntAct dataset. Filter \#1 removed non-human interactors, filter \#2 removed multiple entries with the same experimental setting.

\section{Enrichment Analysis}

GO enrichment analysis has been performed by the use of 2 different web-based applications.

WebGestalt (analysis on $20^{\text {th }}$ August 2014, WebGestalt was last updated on $30^{\text {th }}$ January 2013.) (Wang 2 et al., 2013; Zhang, Kirov \& Snoddy, 2005) http://bioinfo.vanderbilt.edu/webgestalt/ was used with the 3 following parameters: Reference set for enrichment analysis: h.sapiens_genome, Enrichment: GO 4 analysis; Databases: biological process and cellular component. Analysis was performed using 
95 hypergeometric statistics and Bonferroni's correction for multiple testing. Minimum number of genes

96

97

98

99

100

101

102

103

104

105

106

107

108

109

110

\section{Software}

112 Data were stored and analyzed as Excel spreadsheet files. Graphs were prepared by the use of Prism

113 (GraphPad) and Cytoscape (2.6.2, freely available online at http://www.cytoscape.org/) (Saito et al.,

114 2012; Shannon et al., 2003).

115

\section{Results}

\section{LRRK2 interactors: IntAct and BioGRID merged dataset}

118 The IntAct database was queried for LRRK2 (human, Q5S007), and 542 unfiltered annotations were

119 downloaded. After applying filter \#1 to include only human interactions and filter \#2 to remove

120 multiple annotations with similar experimental details in the same publication (see materials and

121 methods), the total number of annotations dropped from 542 to 307. Of these 307 annotations, 278

122 described interactions between LRRK2 and different partners (heterologous interactions) and 29

123 annotations described LRRK2 self-interaction (homologous interaction). 
124 The BioGRID database was similarly queried, and 260 unfiltered annotations were downloaded. After 125 applying filter \#1 and filter \#2 the total number of annotations dropped from 260 to 230; of which 194 126 were heterologous and 36 homologous interactions.

127 Detailed analysis of the PubMed IDs for the heterologous interactions in the IntAct and BioGRID 128 filtered datasets revealed that annotations came from 43 publications in IntAct and 42 publications in

129 BioGRID. However, only 22 publications were identical in both databases. Thus, considering a total of 13063 annotated papers, $35 \%$ of them were annotated by both IntAct and BioGRID; 65\% were contained 131 either in IntAct (21 publications) or BioGRID (20 publications). For this reason the IntAct and the 132 BioGRID datasets for LRRK2 heterologous interactions, after the previously described filtering, were 133 merged to prepare the final dataset for heterologous interactions only (hereafter referred to as "merged 134 dataset"). In the case of publications duplicated between IntAct and BioGRID only one set of annotations was moved to the merge dataset. Discrepancies were found for some of the shared records;

136 in particular, the number of annotations was not necessarily consistent between IntAct and BioGRID,

137

138

139

140

141

\section{2}

143

144

145

146

147

148

149

150

151

152

153

this has been previously reported (Lehne \& Schlitt, 2009) and is likely due to differences in the technical classification of the experiments. To overcome this problem, in the case of shared publications, the record containing the major number of annotations was selected to be moved into the merged dataset; however, for 6 shared publications differences were as such that a merge of the two records was necessary (details of these 6 problematic records and how they were merged are in Supplementary Table1). The final, merged dataset for human LRRK2-human protein interactions (heterologous interactions) consisted of 63 publications describing 422 annotations, captured via different PPI detection methods (Fig.1), for a total of 269 LRRK2 binding partners (here after referred to as the "complete" LRRK2 interactome).

The 422 annotations in the merged dataset were scored based on the following:

Low occurrence - the protein identifier was reported just once in the list of 422 annotations.

Medium-low occurrence - the protein identifier was reported in 1 publication but with 2 or more experimental approaches.

Medium-high occurrence - the protein identifier was reported in 2 or more publications but with the same experimental technique.

High occurrence - the protein identifier was reported in 2 or more publications with 2 or more experimental approaches. 
154 This ranking system allowed us to classify the interactors as follows: 207 interactors were annotated

155 only once; 24 LRRK2 interactors were reported in 1 publication only but with different methods; 13

156 LRRK2 interactors were reported in more than 1 publication but with the same detection method;

157 finally, 25 LRRK2 interactors were reported in more than 1 publication and with different experimental

158 techniques. Interactors annotated only once are detailed in Supplementary Table 2. We designated

159 interactors that were reported with 2 or more annotations to represent the "LRRK2 interactome", this

160 dataset contains 62 proteins (Fig. 2) and is the dataset that was used for enrichment analysis.

161 The 62 proteins in the LRRK2 interactome were segregated into protein family groups according to

162 their UniProtKB record "family\&domains" (http:/www.uniprot.org/uniprot/). 22 of the genes could be

163 assigned to 10 different families: heat shock protein 90 family; tubulin (alpha and beta) family;

164 argonaute family; small GTPase superfamily, Rho family; STE Ser/Thr protein kinase family; 14-3-3

165 family; TRAFAC class dynamin-like GTPase superfamily, dynamin/Fzo/YdjA family; DSH family,

166 small GTPase superfamily, Rab family and actin family (Fig.3).

167

168 GO: "biological process" and "cellular component" enrichment analysis (WebGestalt)

169 The online platform WebGestalt was used to conduct a GO term enrichment analysis for the 62

170 LRRK2 interactors described in Figure 2. The enrichment settings allowed for retrieval of the first

171 top10 hits. The enriched "GO biological process" terms identified were related to transport/localization

172 and cell organization, with a couple of terms supporting an involvement of the LRRK2 interactome in

173 regulating kinase activity (Fig. 4). For the enriched "GO cell component" terms, as expected, the

174 majority of the LRRK2 interactors were annotated as located in the cytoplasm or cytosol-associated

175 components. However, on a more specific level, the interactors were clustered to vesicles, cytoskeleton

176 and cell projections (Fig. 5). The enriched terms were sorted in GO term groups. Groups were

177 identified as cell organization, transport/localization, regulation of kinase activity, cytosol,

178 cytoskeleton, vesicles and cell projections (Supplementary Table 3). The WebGestalt enrichment

179 portal returns the break-down of proteins contributing toward the enrichment of each single term

180 (Supplementary Dataset 1). Therefore LRRK2 interactors were listed as associated to the groups for

181 which they contributed toward enrichment. The lists were then compared to calculate the percentage of

182 proteins in the LRRK2 interactome to contribute to each single GO term group and the percentage of

183 intersection between groups (Fig. 6). Nearly half of the LRRK2 interactors fall in the groups of

184 transport/localization, cytoskeleton and cell organization. Nearly $30 \%$ of them were associated with 
185 vesicles and cell projections. As expected, each of the GO term groups presented with an intersection

186 of around $100 \%$ with the GO term cytosol group. Of more interest is that $50 \%$ of interactors associated

187 with the regulation of kinase activity were shared with vesicles, and $72 \%$ with transport/localization

188 thus suggesting a possible role of LRRK2 in controlling kinase activities related to vesicle/membranes

189 and transport processes. $79 \%$ of interactors associated with vesicles were also associated with

190 transport/localization, thus implying a possible role of LRRK2 in vesicle dynamics related to

191 intracellular transport/trafficking.

192

193 GO: "biological process" enrichment analysis (Panther)

194 The filtered list of 62 LRRK2 interactors, was also used for a second, independent enrichment analysis

195 using the GO Consortium term enrichment service, supported by Panther (Mi, Muruganujan \&

196 Thomas, 2012). The analysis retrieved 150 "GO biological process" enriched terms. These terms were

197 manually divided into 13 groups: general terms, transport/localization, membrane processes, signalling,

198 regulation of catalysis, metabolism, catabolism, regulation of kinase activity, regulation of

199 mitochondrion organization, cell death, development, cell organization and immune response

200 (complete list of terms, divided in groups, with p-values and sample frequencies is reported in

201 Supplementary Table 4). The terms classified within the general terms group were excluded from

202 following analyses because of their lack of specificity; the terms present in the 12 remaining GO term

203 groups were ranked based on their p-values in significance levels from $10^{-16}$ to $10^{-7}$. The contribution

204 of each functional group toward the enrichment in a particular significant level was calculated as

205 follows (function 1):

206

\section{Function 1}

208

209

210

211

$$
\% \text { functional group G in significance level } N=\frac{\sum_{i=1}^{g}(i) * 100}{\sum_{j=1}^{n}(j)}
$$

Where $g$ is the number of terms enriched in the functional group $\mathrm{G}$ within the significance level $\mathrm{N}$; $\mathrm{n}$ is the total number of enriched terms across all the functional groups within the significance level N.

Results are shown in Figure 7. The significant levels $10^{-16}, 10^{-15}, 10^{-14}$ and $10^{-12}$ were only composed of terms belonging to the functional groups of transport/localization, cell organization and membrane 
212 processes. Once these highly significant terms were taken aside, all the other GO term groups started to

213 present their contributions toward the enrichment (in the significant levels from $10^{-11}$ to $10^{-7}$ ).

214

\section{LRRK2 interactome: IntaAct and BioGRID merged dataset for LRRK2 self-interactions}

216 The IntAct database contained 29 annotations for LRRK2 self-interactions (homologous interactions);

217 BioGRID, a total of 36 homologous interactions. Only 7 publications were shared between the two

218 databases therefore, in the total of 31 publications reporting LRRK2 self-interaction, $77 \%$ of the

219 information was captured independently by IntAct or BioGRID. Following the same procedure used

220 for the heterologous interactions, a merged dataset was prepared, with a final count of 31 publications

221 and 53 annotations for LRRK2 self-interaction, based on different experimental methodologies (Fig. $2228)$.

223 The IntAct database (not BioGRID) reports details (when available) on the regions of the LRRK2 224 protein participating in protein interactions. Based on this information, a profile of the LRRK2 225 fragments/residues associated with LRRK2 self-interaction was prepared (Fig. 9). As expected, the 226 catalytic core of LRRK2 was most frequently reported to be associated with LRRK2 dimerization 227 (with a top score of 17 publications), and all the residues tested directly for self-interaction lay in the 228 catalytic core of LRRK2.

\section{LRRK2 interactome and disease}

231 The complete LRRK2 interactome comprised of 269 proteins (the filtered interactome combined with

232 the interactors identified by 1 annotation only) was compared with data from PD and IBD GWAs.

233 Since the associated SNP is only a marker for a genomic locus, not an indication of a specific gene, all 234 the coding open reading frames (ORF), for a total of 156 ORFs in the interval +/- $200 \mathrm{Kbp}$ from the 235 associated SNP, have been taken into account. The positive matches between GWAs hits and LRRK2 236 interactors were recorded (Table 1). Four proteins in the LRRK2 interactome (SNCA, RAB7L1, GAK 237 and MAPT) were found to be candidate proteins in the PD GWA. These proteins were annotated 238 multiple times as LRRK2 interactors from different publications. Among these proteins, SNCA is not 239 only associated with the risk of sporadic PD, it is also a gene whose mutations are responsible for 240 inheritance of familial PD. 1004 ORFs were identified within a $200 \mathrm{Kbp}$ region either side of 241 associated SNPs for IBD and 17 proteins in the LRRK2 interactome were found to be positive matches, 2424 out of 17 were indicated as candidate proteins in the GWA study, the others were just around the 
243 associated SNP; the LRRK2 interactors matching the IBD GWA, with the exception CDC37, were

244 annotated in just 1 publication.

245

\section{Discussion}

247 The implication of LRRK2 in different human diseases has made this protein the centre of interest for 248 many research groups, leading to a large number of functional studies. The breadth of the LRRK2

249 literature is complemented by its depth, with a wide range of different approaches and techniques used 250 in investigations. A PubMed search for LRRK2 retrieved 1257 publications on the 30 $0^{\text {th }}$ October 2014. 251 As a consequence of this, LRRK2 is an excellent candidate for in silico analysis to critically appraise 252 the literature creating a synthesis of our understanding of the LRRK2 interactome. LRRK2 literature 253 can be divided into two different blocks, the first one comprises functional publications that study 254 LRRK2 without reporting PPI data, the second contains a smaller number of publications with details 255 about physical interactions between LRRK2 and partners. This second set of publications is what is 256 annotated in PPI databases. The critical collection of the LRRK2 interacting proteins can be used to 257 generate the state of the art LRRK2 interactome. The interactome can be then analysed to rationalize 258 the understanding of the existing functional literature, which describe the role of LRRK2 in a multitude 259 of biological processes (not annotated in PPI databases). The result of such analysis can also suggest 260 possible future wet-lab investigations.

262 When searching for LRRK2 interactions, we found that the information stored in the IntAct and 263 BioGRID databases overlapped only for around 50\%; this is probably a consequence of the curator's 264 choice in paper selection and the database specialization. Moreover IntAct and BioGRID are both 265 members of the IMEX consortium and thus committed to minimize redundant annotations (Orchard et 266 al., 2012b). Therefore, to have a more detailed view of the LRRK2 interactome the two databases were 267 merged. After filtering out interactions with non-human partners and repeated annotations, 422 entries, 268 corresponding to 269 different interactors, were found to describe the LRRK2 interactome. Only $23 \%$ 269 of the interactors (62 interactors) were confirmed with 2 or more annotations coming from either 270 different publications or different experimental approaches; the percentage was reduced to $9.3 \%(25$ 271 interactors) when the selection criteria were strengthened allowing confirmation in at least 2 different 272 publications and by using at least 2 different techniques. It is noteworthy that LRRK2 is annotated 273 frequently as self-interaction partner. This has been reported in 31 publications and classified with 54 
274 entries. After analysis of the LRRK2 interactors that have been confirmed with at least 2 annotations, $27553 \%$ of the interactors fall into 10 protein families; 8 of those were identified independently by 276 different research groups. This first result has at least 2 consequences. First, it suggests that, based on 277 the features of the annotations stored in databases, not all entries are equal. Future functional research 278 should therefore be oriented toward the interactors with stronger experimental evidence, while more 279 biochemical investigation is needed to test the others, to confirm or discard them. Secondly, by looking 280 at the methods used to retrieve the interactions, it has to be taken into account that many annotations 281 come from hypothesis driven approaches: if a protein was previously mentioned, it is more likely that 282 other researchers will plan to investigate it. This results in a degree of ascertainment bias: when a 283 protein is identified as probable interactor, then it is more frequently examined and therefore annotated. 284 This may influence the gathering of annotations around a specific family. Frequently annotated 285 proteins are probably reliable interaction partners. Many interactors are annotated just once; we have 286 considered them less reliable since they have not yet been confirmed. Some of them may represent 287 false positives; others may have just been ignored by replication studies due to lack of biological 288 appeal with regard to further analysis.

290 The filtered list of 62 LRRK2 interactors (i.e. interactors that have been confirmed with at least 2 291 annotations) was used for enrichment analysis to identify specific biological processes (indicated by 292 GO terms) that LRRK2 is likely to be associated with. These data can be used to infer the function of 293 the LRRK2 interactome. Moreover, based on the assumption that any other protein annotated to these 294 biological processes may interact (directly or indirectly) with LRRK2, these data guide the deduction 295 of additional potential LRRK2 interacting proteins. Enrichment is performed by algorithms developed 296 to recognize if a descriptor (e.g. a GO term associated with biological process or cellular component) is 297 particularly enriched in the list of LRRK2 interactors in comparison with what is expected due to the 298 recurrence of that term in a standard reference list of proteins or genes. This analysis has been 299 performed using Panther (through the GO Consortium portal) and WebGestalt, two freely available 300 online platforms. Since WebGestalt and Panther apply different statistical approaches and use different versions of the GO annotation and ontology datasets they were used to provide 2 independent analyses.

302 The results using both tools were, however, very similar, pointing towards a comparable functional 303 interpretation of LRRK2 interactome; specifically, all the top 10 WebGestalt hits were also present in 304 the Panther enriched list. It has to be noted that a number of 150 genes/proteins is generally suggested 
as minimum to obtain a significant enrichment (http://geneontology.org/faq/what-minimuminformation-include-functional-analysis-paper). Our analysis included just 62 LRRK2 interactors, nevertheless we found statistically significant enrichment with both of the two algorithms we used. This may suggest that the group of proteins we used as input (the LRRK2 interactome) had a low level of background noise which therefore didn't mask enrichment, and this was probably achieved by using a screened interactome composed only by interactors that have been confirmed in at least one replication study.

In the WebGestalt analysis, $50 \%$ of the LRRK2 interactors contributed toward the enrichment of functional terms related with transport/localization, $68 \%$ with cell organization and $29 \%$ with regulation of kinase activity. $40 \%$ of the LRRK2 interactors contributed toward the enrichment of terms associated with the cytoskeleton, $37 \%$ with cell projections and $31 \%$ with vesicles. The enrichment of terms related to the regulation of kinase activity was expected, as LRRK2 is an active kinase and likely to regulate other kinases in cascade. However the LRRK2 interactors contributing to this specific enrichment were also present in the GO term groups of vesicles $(50 \%)$ and transport/localization (72\%) thus suggesting LRRK2's interactome may regulate kinase functions in vesicle transport. $60 \%$ of the LRRK2 interactors involved in the GO term group of cytoskeleton also contributed toward the enrichment of transport. Similarly, 79\% of the interactors involved in the GO term group of vesicles were also annotated as involved in transport, thus suggesting that the LRRK2 interactome influences transport processes related with cytoskeleton and vesicles dynamics. These results are in accordance with recent functional literature that sees a role of LRRK2 in vesicle mediated processes like endocytosis (Alegre-Abarrategui et al., 2009), synaptic function (Cirnaru et al., 2014; Parisiadou et al., 2014) and autophagy (Schapansky et al., 2014; Manzoni et al., 2013; Gómez-Suaga et al., 2012). It is of note that the papers referenced in this discussion section are not annotated in the BioGRID neither in the IntAct databases since they are either too recent, or they report only a functional evidence for LRRK2 activity and do not contain PPI records. For this reason they have not contributed toward the enrichment, so they independently support the results.

The Panther analysis identified more descriptive GO terms as enriched, compared with the WebGestalt analysis (supplementary Table 4). For this reason, we then analysed details of each single, enriched GO term group. For the most significantly enriched group in Panther, i.e transport/localization, the 3 most 
336

337

338

339

340

341

342

343

344

345

346

347

348

349

350

351

352

353

354

355

356

357

358

359

360

361

362

363

364

365

specific terms were "cytoskeleton-dependent intracellular transport", "cellular protein localization" and "vesicle-mediated transport" thus reiterating the importance of LRRK2 interactome for the process of transport and vesicular trafficking. GTPases and regulators of GTPase activity are a protein group represented in the LRRK2 interactome, it is therefore unsurprising that the GO term GTP catabolic process was enriched in the analysis. Similarly, the enrichment of many terms related to nucleotide catabolism and metabolism was expected due to the presence of ATP/GTPases in the LRRK2 interactome. The function of many kinases is to regulate other kinases therefore the enrichment of terms associated with kinase regulation was expected. It was however impossible to predict from this analysis whether the 11 LRRK2 associated kinases (Supplementary Table 5) are being regulated by LRRK2 or whether the LRRK2 interactome is regulating LRRK2's activity. The child terms in the GO term group of cell death specifically indicated the regulation of this process and the special cell death defined as apoptosis. Very interestingly, 3 out of the 5 terms enriched in the GO domain of regulation of mitochondrion organization were related to the regulation of mitochondrial processes in apoptosis (supplementary Table 4). The combination of this information may suggest that the key enriched process is apoptosis rather than the general process of cell death, and that it is the apoptotic processes taking place at the mitochondria, rather than mitochondria generic processes, which are relevant to the function of the LRRK2 interactome. When the signalling processes were looked at in detail, all the enriched terms appeared to be very general, with the exception of "Fc receptor signalling pathway" (pvalue $=1.32 \mathrm{e}^{-11}, 21 \%$ of LRRK2 interactors contributing to the enrichment of this term). This is a very specific GO term that in the ancestor GO chart is directly connected with cell surface signaling and immune response, and supports the recent findings that microglial Fc receptors have been associated with alpha-synuclein-induced pro-inflammatory signaling in PD (Cao, Standaert \& Harms, 2012). There were 8 terms enriched within the immune response domain; 4 of them were parents of the specific term "immune response-regulating cell surface receptor signaling pathway" and thus also have a parental relationship with the enriched GO term "Fc receptor signaling pathway". It is known that LRRK2 is expressed in the immune system (Thévenet et al., 2011) and that its total level and phosphorylation state are modulated by immune cell stimulation (Moehle et al., 2012; Kubo et al., 2010). Finally, the developmental process domain presented with 15 enriched terms; 5 of them were general terms; the remaining 10 terms were associated with development of neurons and the nervous system. Very little is known about LRRK2 and development; however it has been shown that total 
366 LRRK2 levels vary according to different developmental stages (Giesert et al., 2013; Zechel et al., 367 2010).

In the light of these observations we propose the function of LRRK2 interactome to be associated with transport and trafficking, possibly regulating enzymatic events associated with cytoskeleton and vesicles. However, we recognize that LRRK2 probably has a role as a hub protein, thus controlling many different functions at a time, and this is likely to be one of the reasons why it has proved so challenging to reach a consensus on LRRK2 physiological function. Based on our results we suggest the role of LRRK2 can also be modulated depending on the cell type analysed (i.e. immune versus nervous system) and possibly the developmental stage, so the outcome of wet lab research may depend on the cellular system used to infer interaction. Not only does this give a possible explanation for the number of different functions that are described for LRRK2 in different experimental models; it also suggests a possible reason for the plethora of different diseases LRRK2 is associated with and opens to discussions of a LRRK2-oriented therapy. If LRRK2 regulates different biological processes in different tissues, a hypothetical targeting of LRRK2 during disease may generate unexpected, and unwanted, side effects. It would be beneficial to analyse LRRK2 interactome according on the cell type in which the interaction was reported. Unfortunately at the moment the majority of the annotations comes from in vitro studies and cancer cell lines; we don't have enough annotations from primary cultures and physiological model systems to perform cell type specific analysis yet. The analysis of the $200 \mathrm{Kbp}$ around the associated SNPs for PD and IBD revealed that some of the proteins encoded in these regions are included in the LRRK2 interactome. The influence of associated SNPs on the control of the regions around them is unknown. They are markers for loci involved in the risk for disease, they may modify the gene in which they actually reside or alternatively regulate multiple genes around them. The presence of 21 LRRK2 interactors around associated SNPs for PD and IBD reinforces the role of LRRK2 in these diseases and provides a system to prioritise candidates to be evaluated in the context of the molecular mechanism of sporadic PD and IBD.

\section{Conclusions}

The analysis of the LRRK2 interactome we described above provides an overview of the published research on this protein, and a system in which to identify the interacting proteins with the most reliable supporting experimental evidence. The enrichment analysis of this interactome provides an 
397 indication of the pathways and processes LRRK2 can influence within its cellular environment. This 398 analysis indicates that LRRK2 may be involved in more than one function, perhaps depending on the 399 cellular type and developmental stage. Intriguingly, some of the proteins in the LRRK2 interactome

400 have also been suggested to be genetic risk factors for PD and IBD. Our approach can therefore guide 401 future research priorities as it suggests focusing on these interactors and their relationship to LRRK2 402 and disease. Moreover the multiple interactions and cellular functions associated with LRRK2 suggest 403 caution in using it as a drug target given the potential for off-target problems. Importantly, in silico 404 analyses such as those described above can provide a starting point for further hypothesis driven wet 405 laboratory based investigations - opening up new avenues that may reveal important insights into the 406 biology of LRRK2.

407

408 References

409 Alegre-Abarrategui J, Christian H, Lufino MM, Mutihac R, Venda LL, Ansorge O, Wade-Martins R. 410 2009. LRRK2 regulates autophagic activity and localizes to specific membrane microdomains in a 411 novel human genomic reporter cellular model. Human Molecular Genetics 18:4022-4034.

413 Ashburner M, Ball CA, Blake JA, Botstein D, Butler H, Cherry JM, Davis AP, Dolinski K, Dwight SS, 414 Eppig JT, Harris MA, Hill DP, Issel-Tarver L, Kasarskis A, Lewis S, Matese JC, Richardson JE, 415 Ringwald M, Rubin GM, Sherlock G. 2000. Gene ontology: tool for the unification of biology. The 416 Gene Ontology Consortium. Nature Genetics 25:25-29

418 Breitkreutz BJ, Stark C, Tyers M. 2003. The GRID: the General Repository for Interaction Datasets. 419 Genome Biology 4: R23.

421 Cao S, Standaert DG, Harms AS. 2012. The gamma chain subunit of Fc receptors is required for alpha422 synuclein-induced pro-inflammatory signaling in microglia. Journal of Neuroinflammation 9:259.

424 Chatr-Aryamontri A, Breitkreutz BJ, Heinicke S, Boucher L, Winter A, Stark C, Nixon J, Ramage L, 425 Kolas N, O'Donnell L, Reguly T, Breitkreutz A, Sellam A, Chen D, Chang C, Rust J, Livstone M, 426 Oughtred R, Dolinski K, Tyers M. 2013. The BioGRID interaction database: 2013 update. Nucleic 427 Acids Research 41: D816-23. 
429 Cirnaru MD, Marte A, Belluzzi E, Russo I, Gabrielli M, Longo F, Arcuri L, Murru L, Bubacco L, 430 Matteoli M, Fedele E, Sala C, Passafaro M, Morari M, Greggio E, Onofri F, Piccoli G. 2014. LRRK2 431 kinase activity regulates synaptic vesicle trafficking and neurotransmitter release through modulation 432 of LRRK2 macro-molecular complex. Frontiers in Molecular Neuroscience 7:49.

433

434 435 436 437 438 439 440 441 442

Dzamko N, Deak M, Hentati F, Reith AD, Prescott AR, Alessi DR, Nichols RJ. 2010. Inhibition of LRRK2 kinase activity leads to dephosphorylation of Ser(910)/Ser(935), disruption of 14-3-3 binding and altered cytoplasmic localization. Biochemical Journal 430: 405-413.

Do CB, Tung JY, Dorfman E, Kiefer AK, Drabant EM, Francke U, Mountain JL, Goldman SM, Tanner CM, Langston JW, Wojcicki A, Eriksson N. 2011. Web-based genome-wide association study identifies two novel loci and a substantial genetic component for Parkinson's disease. PLoS Genetics 7:e1002141.

Giesert F, Hofmann A, Bürger A, Zerle J, Kloos K, Hafen U, Ernst L, Zhang J, Vogt-Weisenhorn DM, Wurst W. 2013. Expression analysis of Lrrk1, Lrrk2 and Lrrk2 splice variants in mice. PLoS One 8:e63778.

Gómez-Suaga P, Luzón-Toro B, Churamani D, Zhang L, Bloor-Young D, Patel S, Woodman PG, Churchill GC, Hilfiker S. 2012. Leucine-rich repeat kinase 2 regulates autophagy through a calciumdependent pathway involving NAADP. Human Molecular Genetics 21:511-525.

Greggio E, Zambrano I, Kaganovich A, Beilina A, Taymans JM, Daniëls V, Lewis P, Jain S, Ding J, Syed A, Thomas KJ, Baekelandt V, Cookson MR. 2008. The Parkinson disease-associated leucine-rich repeat kinase 2 (LRRK2) is a dimer that undergoes intramolecular autophosphorylation. Journal Biological Chemistry 283:16906-16914.

Hermjakob H, Montecchi-Palazzi L, Lewington C, Mudali S, Kerrien S, Orchard S, Vingron M, Roechert B, Roepstorff P, Valencia A, Margalit H, Armstrong J, Bairoch A, Cesareni G, Sherman D, 
458 Apweiler R. 2004. IntAct: an open source molecular interaction database. Nucleic Acids Research 32:

459

460

461

462

463

464

465

466

467

468

469

470

471

472

473

474

475

476

477

478

479

480

481

482

483

484

485

486

487

D452-5.

International Parkinson's Disease Genomics Consortium (IPDGC), Wellcome Trust Case Control

Consortium 2 (WTCCC2). 2011. A two-stage meta-analysis identifies several new loci for Parkinson's disease. PLoS Genetics 7: e1002142.

Inzelberg R, Cohen OS, Aharon-Peretz J, Schlesinger I, Gershoni-Baruch R, Djaldetti R, Nitsan Z, Ephraty L, Tunkel O, Kozlova E, Inzelberg L, Kaplan N, Fixler Mehr T, Mory A, Dagan E,

Schechtman E, Friedman E, Hassin-Baer S. 2012. The LRRK2 G2019S mutation is associated with Parkinson disease and concomitant non-skin cancers. Neurology 78:781-786.

Jostins L, Ripke S, Weersma RK, Duerr RH, McGovern DP, Hui KY, Lee JC, Schumm LP, Sharma Y, Anderson CA, Essers J, Mitrovic M, Ning K, Cleynen I, Theatre E, Spain SL, Raychaudhuri S, Goyette P, Wei Z, Abraham C, Achkar JP, Ahmad T, Amininejad L, Ananthakrishnan AN, Andersen V, Andrews JM, Baidoo L, Balschun T, Bampton PA, Bitton A, Boucher G, Brand S, Büning C, Cohain A, Cichon S, D'Amato M, De Jong D, Devaney KL, Dubinsky M, Edwards C, Ellinghaus D, Ferguson LR, Franchimont D, Fransen K, Gearry R, Georges M, Gieger C, Glas J, Haritunians T, Hart A, Hawkey C, Hedl M, Hu X, Karlsen TH, Kupcinskas L, Kugathasan S, Latiano A, Laukens D, Lawrance IC, Lees CW, Louis E, Mahy G, Mansfield J, Morgan AR, Mowat C, Newman W, Palmieri O, Ponsioen CY, Potocnik U, Prescott NJ, Regueiro M, Rotter JI, Russell RK, Sanderson JD, Sans M, Satsangi J, Schreiber S, Simms LA, Sventoraityte J, Targan SR, Taylor KD, Tremelling M, Verspaget HW, De Vos M, Wijmenga C, Wilson DC, Winkelmann J, Xavier RJ, Zeissig S, Zhang B, Zhang CK, Zhao H; International IBD Genetics Consortium (IIBDGC), Silverberg MS, Annese V. Hakonarson H, Brant SR, Radford-Smith G, Mathew CG, Rioux JD, Schadt EE, Daly MJ, Franke A, Parkes M, Vermeire S, Barrett JC, Cho JH. 2012. Host-microbe interactions have shaped the genetic architecture of inflammatory bowel disease. Nature 491:119-124.

Kerrien S, Alam-Faruque Y, Aranda B, Bancarz I, Bridge A, Derow C, Dimmer E, Feuermann M, Friedrichsen A, Huntley R, Kohler C, Khadake J, Leroy C, Liban A, Lieftink C, Montecchi-Palazzi L, 
488 Orchard S, Risse J, Robbe K, Roechert B, Thorneycroft D, Zhang Y, Apweiler R, Hermjakob H. 2007. 489 IntAct - an open source molecular interaction database. Nucleic Acids Research 35: D561-5.

490

491 Kubo M, Kamiya Y, Nagashima R, Maekawa T, Eshima K, Azuma S, Ohta E, Obata F. 2010. LRRK2

492 is expressed in B-2 but not in B-1 B cells, and downregulated by cellular activation. Journal

493 Neuroimmunology 229: 123-128.

494

495 Lehne B, Schlitt T. 2009. Protein-protein interaction databases: keeping up with growing interactomes.

496 Human Genomics 3:291-297.

497

498 Lewis PA, Manzoni C. 2012. LRRK2 and human disease: a complicated question or a question of 499 complexes? Science Signaling 5:pe2.

500

501 Manzoni C, Mamais A, Dihanich S, Abeti R, Soutar MP, Plun-Favreau H, Giunti P, Tooze SA, 502 Bandopadhyay R, Lewis PA. 2013. Inhibition of LRRK2 kinase activity stimulates macroautophagy.

503 Biochimica Biophysica Acta 1833:2900-2910.

504

505 Marcinek P, Jha AN, Shinde V, Sundaramoorthy A, Rajkumar R, Suryadevara NC, Neela SK, van 506 Tong H, Balachander V, Valluri VL, Thangaraj K, Velavan TP. 2013. LRRK2 and RIPK2 variants in 507 the NOD 2-mediated signaling pathway are associated with susceptibility to Mycobacterium leprae in 508 Indian populations. PLoS One 8:e73103.

509

510 Mi H, Muruganujan A, Thomas PD. 2012. PANTHER in 2013: modeling the evolution of gene

511 function, and other gene attributes, in the context of phylogenetic trees. Nucleic Acids Research 41:

512 D377-86.

513

514 Moehle MS, Webber PJ, Tse T, Sukar N, Standaert DG, DeSilva TM, Cowell RM, West AB. 2012.

515 LRRK2 inhibition attenuates microglial inflammatory responses. Journal Neuroscience 32:1602-1611. 516

517 Nalls MA, Pankratz N, Lill CM, Do CB, Hernandez DG, Saad M, DeStefano AL, Kara E, Bras J, 518 Sharma M, Schulte C, Keller MF, Arepalli S, Letson C, Edsall C, Stefansson H, Liu X, Pliner H, Lee 
519 JH, Cheng R, International Parkinson's Disease Genomics Consortium (IPDGC), Parkinson's Study 520 Group (PSG) Parkinson's Research: The Organized GENetics Initiative (PROGENI), 23andMe, 521 GenePD, NeuroGenetics Research Consortium (NGRC), Hussman Institute of Human Genomics 522 (HIHG), Ashkenazi Jewish Dataset Investigator, Cohorts for Health and Aging Research in Genetic 523 Epidemiology (CHARGE), North American Brain Expression Consortium (NABEC), United Kingdom 524 Brain Expression Consortium (UKBEC), Greek Parkinson's Disease Consortium, Alzheimer Genetic 525 Analysis Group, Ikram MA, Ioannidis JP, Hadjigeorgiou GM, Bis JC, Martinez M, Perlmutter JS, 526 Goate A, Marder K, Fiske B, Sutherland M, Xiromerisiou G, Myers RH, Clark LN, Stefansson K, 527 Hardy JA, Heutink P, Chen H, Wood NW, Houlden H, Payami H, Brice A, Scott WK, Gasser T, 528 Bertram L, Eriksson N, Foroud T, Singleton AB. 2014. Large-scale meta-analysis of genome-wide 529 association data identifies six new risk loci for Parkinson's disease. Nature Genetics 46:989-993. 530

531 Orchard S, Kerrien S, Abbani S, Aranda B, Bhate J, Bidwell S, Bridge A, Briganti L, Brinkman FSL, 532 Cesareni G, Chatr-aryamontri A, Chautard E, Chen C, Dumousseau M, Goll J, Hancock REW, 533 Hannick LI, Jurisica I, Khadake J, Lynn DJ, Mahadevan U, Perfetto L, Raghunath A, Ricard-Blum S, 534 Roechert B, Salwinski L, Stümpflen V, Tyers M, Uetz P, Xenarios I, Hermjakob H. 2012. Protein 535 interaction data curation: the International Molecular Exchange (IMEx) consortium. Nature Methods 9: 536 345-350.

538 Paisán-Ruíz C, Jain S, Evans EW, Gilks WP, Simón J, van der Brug M, López de Munain A, Aparicio 539 S, Gil AM, Khan N, Johnson J, Martinez JR, Nicholl D, Carrera IM, Pena AS, de Silva R, Lees A, 540 Martí-Massó JF, Pérez-Tur J, Wood NW, Singleton AB. 2004. Cloning of the gene containing 541 mutations that cause PARK8-linked Parkinson's disease. Neuron 44: 595-600.

543 Paisán-Ruiz C, Lewis PA, Singleton AB. 2013. LRRK2: cause, risk, and mechanism. Journal of 544 Parkinsons Disease 3:85-103.

546 Parisiadou L, Yu J, Sgobio C, Xie C, Liu G, Sun L, Gu XL, Lin X, Crowley NA, Lovinger DM, Cai H. 547 2014. LRRK2 regulates synaptogenesis and dopamine receptor activation through modulation of PKA 548 activity. Nature Neuroscience 17:367-376. 
550 Ruiz-Martínez J, de la Riva P, Rodríguez-Oroz MC, Mondragón Rezola E, Bergareche A, Gorostidi A, 551 Gago B, Estanga A, Larrañaga N, Sarasqueta C, López de Munain A, Martí Massó JF. 2014.

552 Prevalence of cancer in Parkinson's disease related to R1441G and G2019S mutations in LRRK2.

553 Movement Disorders 29:750-755.

554

555 Saito R, Smoot ME, Ono K, Ruscheinski J, Wang PL, Lotia S, Pico AR, Bader GD, Ideker T. 2012. A 556 travel guide to Cytoscape plugins. Nature Methods 9:1069-1076.

557

558

Saunders-Pullman R, Barrett MJ, Stanley KM, Luciano MS, Shanker V, Severt L, Hunt A, Raymond

560 D, Ozelius LJ, Bressman SB. 2010. LRRK2 G2019S mutations are associated with an increased cancer

561

562 risk in Parkinson disease. Movement Disorders 25: 2536-2541.

\section{3}

564

565

566

567

568

569

570

571 572

573

574 575

576

577 578

579 580

Schapansky J, Nardozzi JD, Felizia F, LaVoie MJ. 2014. Membrane recruitment of endogenous LRRK2 precedes its potent regulation of autophagy. Human Molecular Genetics 15: 4201-4214.

Shannon P, Markiel A, Ozier O, Baliga NS, Wang JT, Ramage D, Amin N, Schwikowski B, Ideker T. 2003. Cytoscape: a software environment for integrated models of biomolecular interaction networks. Genome Research 13:2498-2504.

Taymans JM, Vancraenenbroeck R, Ollikainen P, Beilina A, Lobbestael E, De Maeyer M, Baekelandt V, Cookson MR. 2011. LRRK2 kinase activity is dependent on LRRK2 GTP binding capacity but independent of LRRK2 GTP binding. PLoS One 6:e23207.

Thévenet J, Pescini Gobert R, Hooft van Huijsduijnen R, Wiessner C, Sagot YJ. 2011. Regulation of LRRK2 expression points to a functional role in human monocyte maturation. PLoS One 6:e21519.

Wang J, Duncan D, Shi Z, Zhang B. 2013. WEB-based GEne SeT AnaLysis Toolkit (WebGestalt): update 2013. Nucleic Acids Research 41:W77-83.

Zhang B, Kirov S, Snoddy J. 2005. WebGestalt: an integrated system for exploring gene sets in various biological contexts. Nucleic Acids Research 33:W741-8. 
582 Zhang FR, Huang W, Chen SM, Sun LD, Liu H, Li Y, Cui Y, Yan XX, Yang HT, Yang RD, Chu TS, 583 Zhang C, Zhang L, Han JW, Yu GQ, Quan C, Yu YX, Zhang Z, Shi BQ, Zhang LH, Cheng H, Wang 584 CY, Lin Y, Zheng HF, Fu XA, Zuo XB, Wang Q, Long H, Sun YP, Cheng YL, Tian HQ, Zhou FS, Liu 585 HX, Lu WS, He SM, Du WL, Shen M, Jin QY, Wang Y, Low HQ, Erwin T, Yang NH, Li JY, Zhao X, 586 Jiao YL, Mao LG, Yin G, Jiang ZX, Wang XD, Yu JP, Hu ZH, Gong CH, Liu YQ, Liu RY, Wang 587 DM, Wei D, Liu JX, Cao WK, Cao HZ, Li YP, Yan WG, Wei SY, Wang KJ, Hibberd ML, Yang S, 588 Zhang XJ, Liu JJ. 2009. Genomewide association study of leprosy. New England Journal of Medicine $589 \quad 361: 2609-2618$.

590

591 Zhang F, Liu H, Chen S, Low H, Sun L, Cui Y, Chu T, Li Y, Fu X, Yu Y, Yu G, Shi B, Tian H, Liu D, 592 Yu X, Li J, Lu N, Bao F, Yuan C, Liu J, Liu H, Zhang L, Sun Y, Chen M, Yang Q, Yang H, Yang R, 593 Zhang L, Wang Q, Liu H, Zuo F, Zhang H, Khor CC, Hibberd ML, Yang S, Liu J, Zhang X. 2011. 594 Identification of two new loci at IL23R and RAB32 that influence susceptibility to leprosy. Nature 595 Genetics 43:1247-1251.

596

597 Zechel S, Meinhardt A, Unsicker K, von Bohlen Und Halbach O. 2010. Expression of leucine-rich598 repeat-kinase 2 (LRRK2) during embryonic development. International Journal of Developmental 599 Neuroscience 28:391-399.

600

601 Zimprich A, Biskup S, Leitner P, Lichtner P, Farrer M, Lincoln S, Kachergus J, Hulihan M, Uitti RJ, 602 Calne DB, Stoessl AJ, Pfeiffer RF, Patenge N, Carbajal IC, Vieregge P, Asmus F, Müller-Myhsok B, 603 Dickson DW, Meitinger T, Strom TM, Wszolek ZK, Gasser T. 2004. Mutations in LRRK2 cause 604 autosomal-dominant parkinsonism with pleomorphic pathology. Neuron 44:601-607. 605 


\section{1}

LRRK2 heterologous interactions as reported in the merged dataset.

LRRK2 in the middle of the graph is linked to candidate partners (black dots) through 422 annotations as described in the merged dataset. Some partners have one connection only; others have multiple connections based on the number of annotations. Different colours represent different methods used to infer the interaction; note that since IntAct and BioGRID use different classifications for the interaction detection method, a simplified and harmonized version has been applied to this figure to help the reader. In particular: i) Affinity Capture stands for - Affinity Capture-Western and Affinity Capture-MS (in BioGRID) - Anti Bait Coimmunoprecipitation, Anti Tag Coimmunoprecipitation, Coimmunoprecipitation, Pull Down and Affinity Chromatography Technology (in IntAct); ii) Biochemical Activity stands for Biochemical Activity (in BioGRID) - Protein Kinase Assay (in IntAct); iii) Co-localization stands for - Co-localization (in BioGRID) - Confocal microscopy and Fluorescence Microscopy (in IntAct); iv) Reconstituted Complex stands for - Fluorescence Polarization Spectroscopy and Surface Plasmon Resonance (in IntAct). 


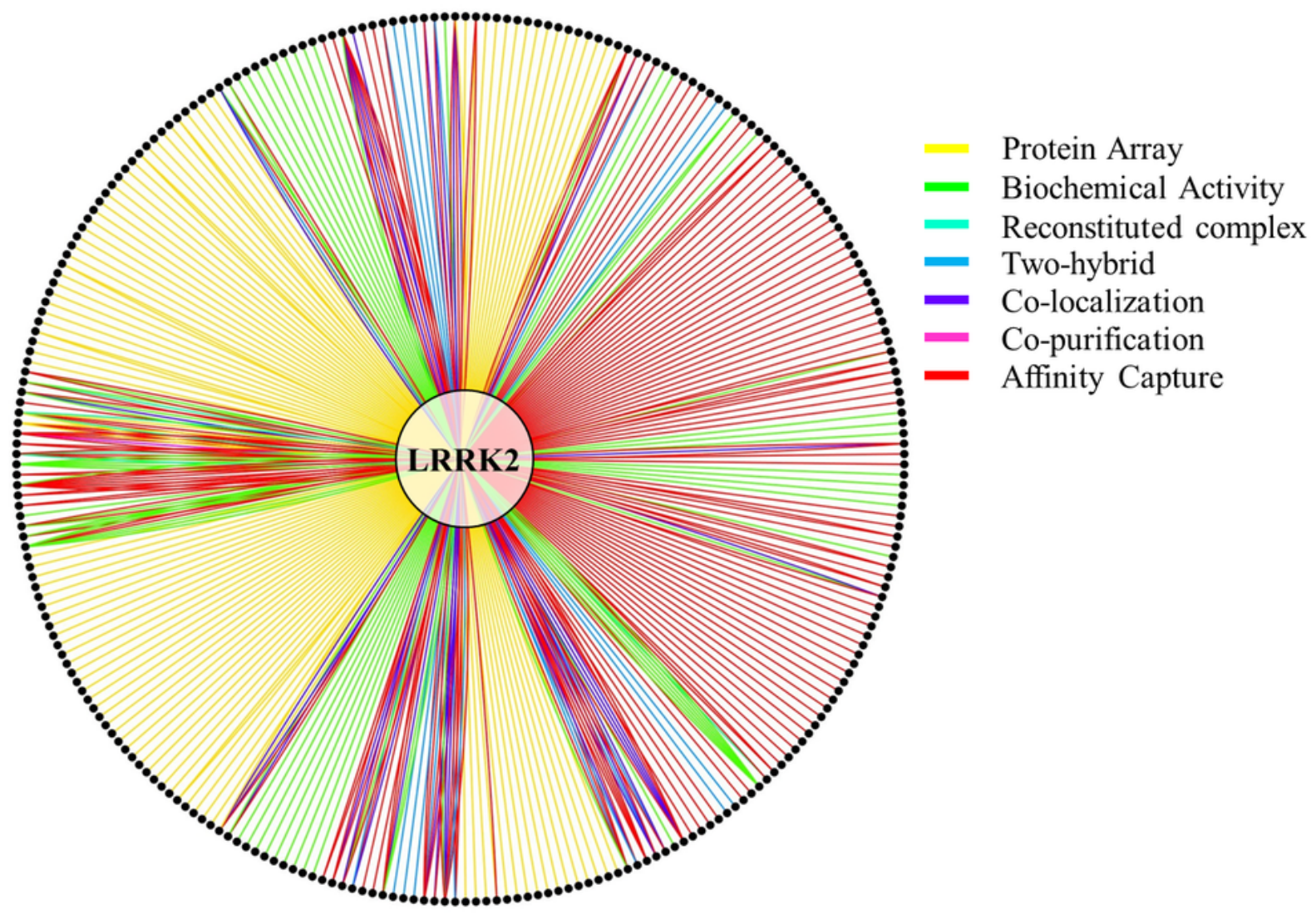




\section{2}

Number of annotations capturing each of the LRRK2 interactors.

Only the interactors reported in 2 or more annotations, and used in the enrichment analysis, are included in the figure, all other interactors were identified in just a single experiment. A) High occurrence LRRK2 interactors, >1 publication, >1 method; B) Medium-high occurrence LRRK2 interactors, >1 publication, 1 method; and C) Medium-low occurrence LRRK2 interactors, 1 publication, $>1$ method.

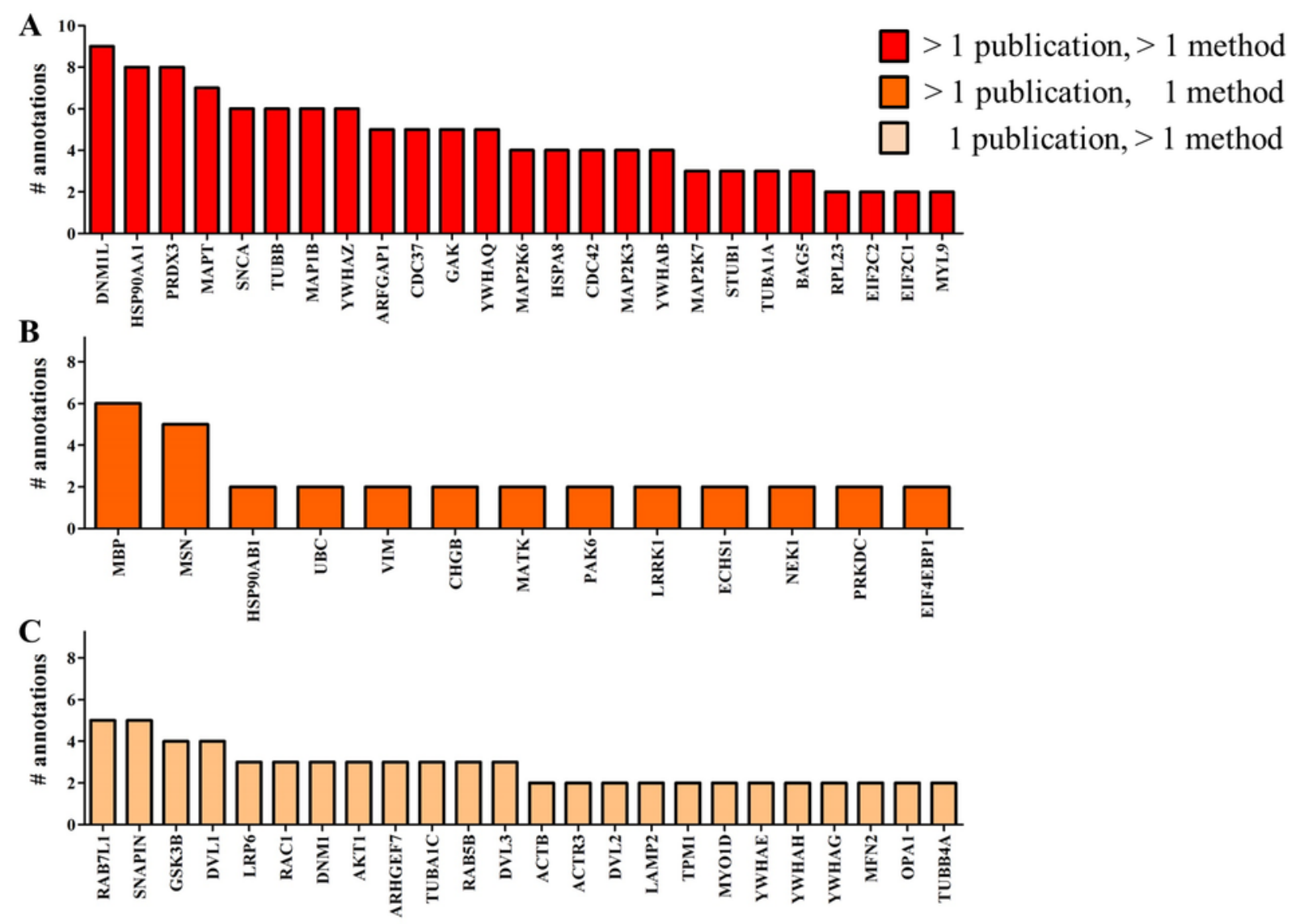




\section{3}

Families of LRRK2 interactors.

Each of the 62 proteins in the LRRK2 filtered interactome was associated to a family (if available) based on the UniProtKB "family\&domains" classification. Proteins belonging to the same family were plotted accordingly to the total number of publications ( $y$-axis); each family was then classified based on the authorships as non-independent (different family members described by the same research group) or independent (different family members described by at least two different research groups).

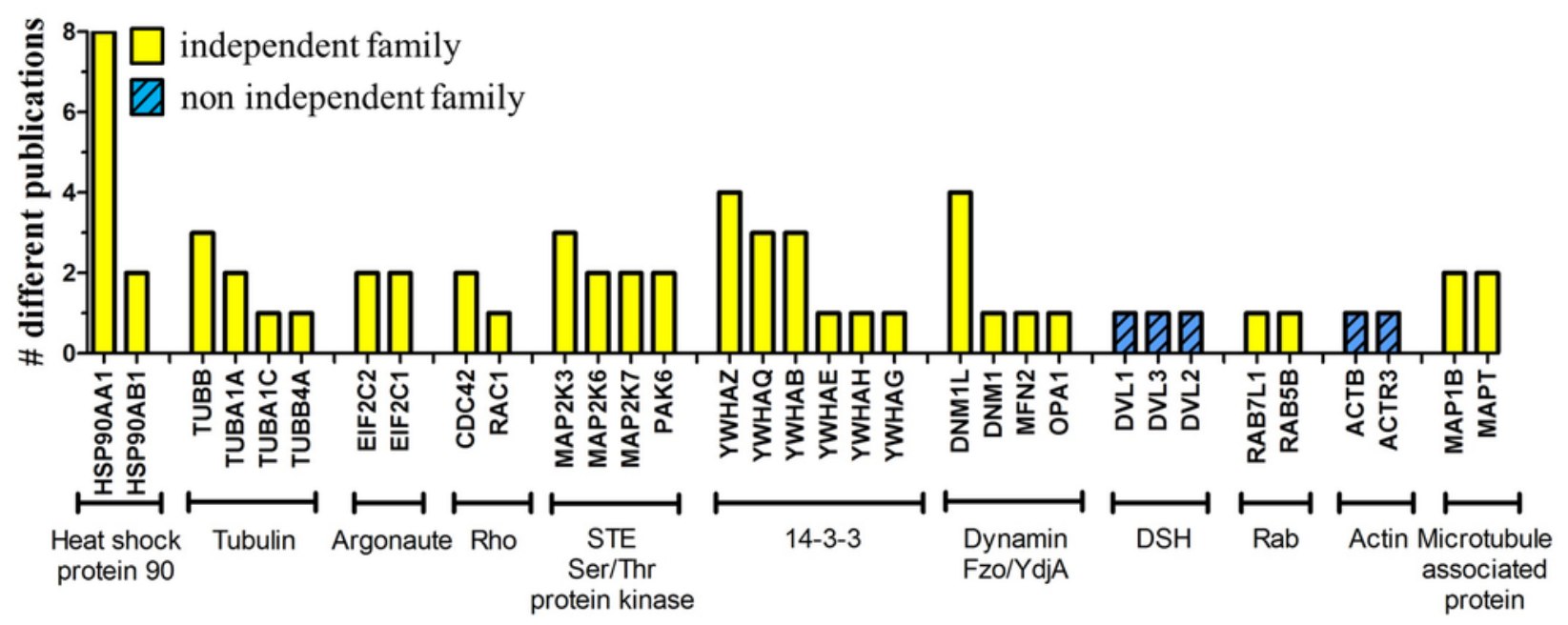




\section{4}

Dendrogram of "GO biological process" terms enriched for LRRK2 interactors in WebGestalt.

Hierarchical levels of the dendrogram are alternatively represented in blue and yellow; red

text indicates the top $10 \mathrm{GO}$ terms. The table lists details of the enriched terms: GO term and ID, number of proteins in the GO term category (Ref.), number of LRRK2 interactors associated with the GO term (Genes), p-value adjusted for multiple testing. 


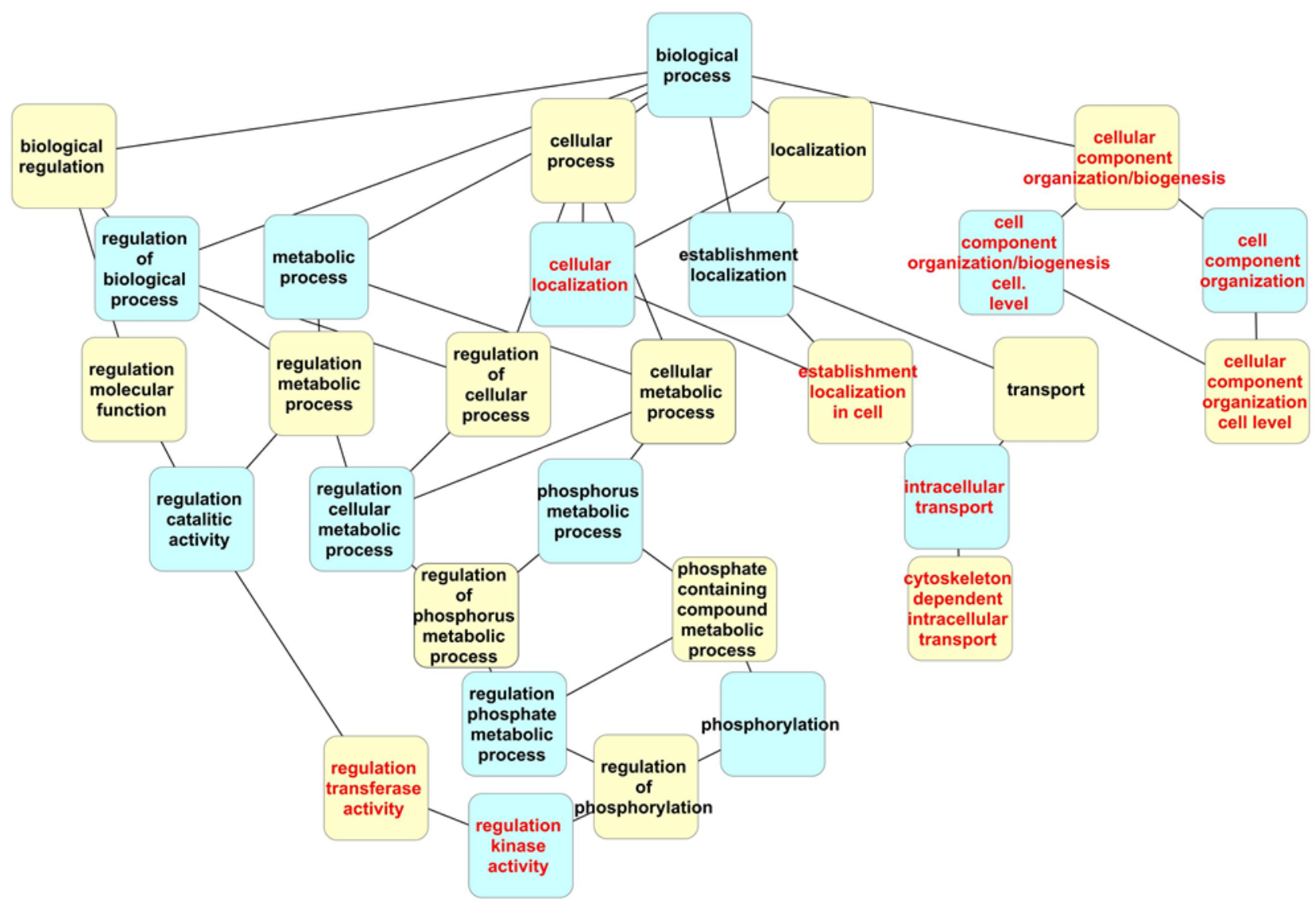

\begin{tabular}{|l|l|l|l|c|}
\hline \multicolumn{1}{|c|}{ Biological Process GO term } & GO ID. & Ref. & Genes & Adjusted p-value \\
\hline Cellular localization & GO:0051641 & 1977 & 31 & $3.80 \mathrm{e}^{-09}$ \\
\hline Intracellular transport & GO:0046907 & 1131 & 24 & $6.72 \mathrm{e}^{-09}$ \\
\hline $\begin{array}{l}\text { Cellular component organization } \\
\text { at cellular level }\end{array}$ & GO:0071842 & 3317 & 38 & $3.93 \mathrm{e}^{-08}$ \\
\hline Cellular component organization & GO:0016043 & 4105 & 42 & $4.55 \mathrm{e}^{-08}$ \\
\hline Establishment localization in cell & GO:0051649 & 1756 & 28 & $5.38 \mathrm{e}^{-08}$ \\
\hline Regulation of kinase activity & GO:0043549 & 632 & 18 & $5.55 \mathrm{e}^{-08}$ \\
\hline Regulation of transferase activity & GO:0051338 & 653 & 18 & $9.48 \mathrm{e}^{-08}$ \\
\hline $\begin{array}{l}\text { Cellular component organization } \\
\text { or biogenesis at cellular level }\end{array}$ & GO:0071841 & 3420 & 38 & $1.03 \mathrm{e}^{-07}$ \\
\hline $\begin{array}{l}\text { Cellular component organization } \\
\text { or biogenesis }\end{array}$ & GO:0071840 & 4222 & 42 & $1.21 \mathrm{e}^{-07}$ \\
\hline $\begin{array}{l}\text { Cytoskeleton-dependent } \\
\text { intracellular transport }\end{array}$ & GO:0030705 & 66 & 8 & $3.01 \mathrm{e}^{-07}$ \\
\hline
\end{tabular}




\section{5}

Dendrogram of "GO cell component" terms enriched for LRRK2 interactors in WebGestalt.

Hierarchical levels of the dendrogram are alternatively represented in blue and yellow; red

text indicates the top $10 \mathrm{GO}$ terms. The table lists details of the enriched terms: GO term and ID, number of proteins in the GO term category (Ref.), number of LRRK2 interactors associated with the GO term (Genes), p-value adjusted for multiple testing. 


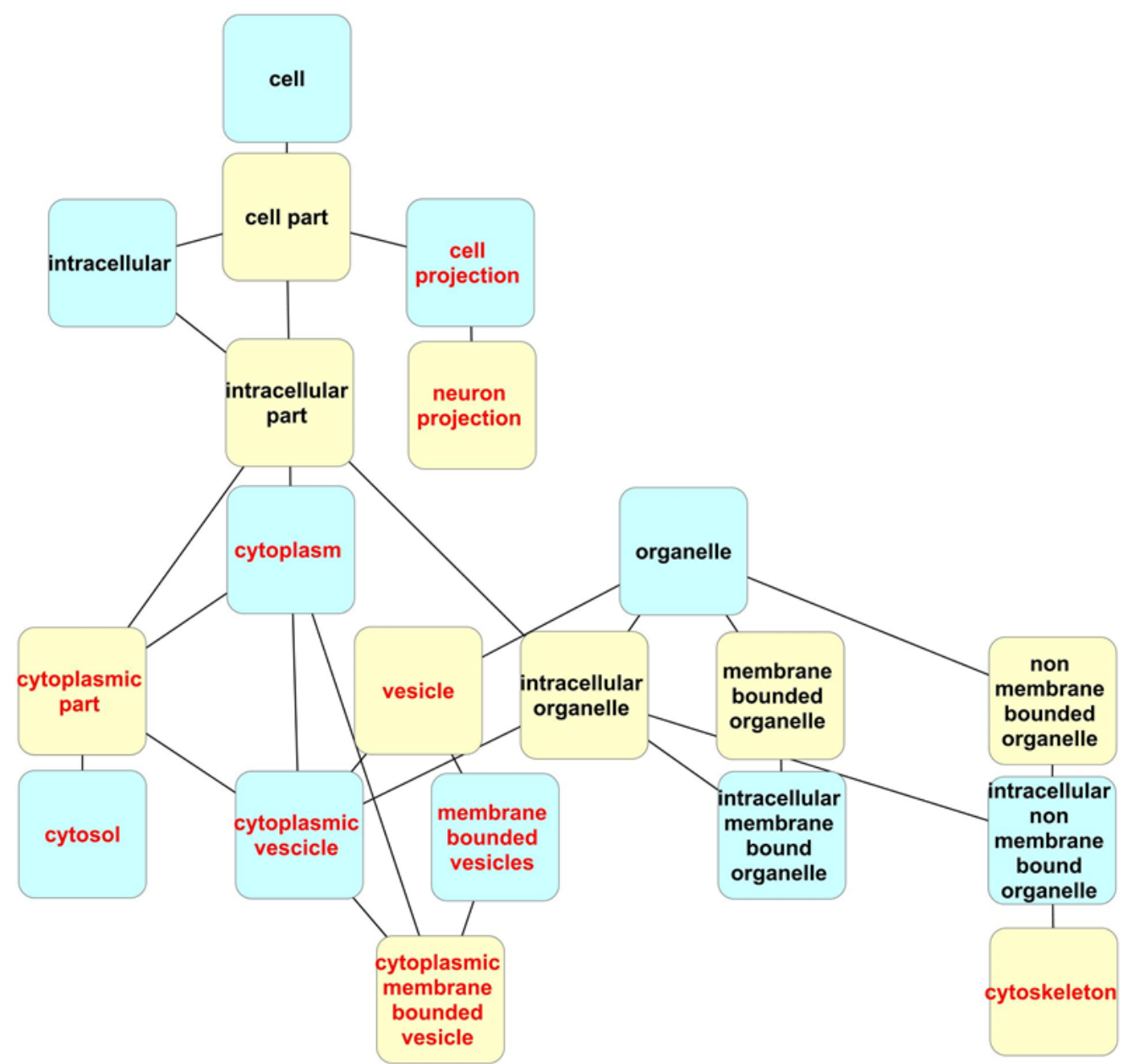

\begin{tabular}{|l|l|l|l|c|}
\hline \multicolumn{1}{|c|}{ Cellular Component GO term } & GO ID & Ref. & Genes & Adjusted p-value \\
\hline cytosol & GO:0005829 & 2372 & 40 & $8.60 \mathrm{e}^{-18}$ \\
\hline cytoplasmic part & GO:0044444 & 6772 & 56 & $1.41 \mathrm{e}^{-14}$ \\
\hline cytoplasm & GO:0005737 & 9130 & 59 & $5.29 \mathrm{e}^{-11}$ \\
\hline cell projection & GO:0042995 & 1230 & 23 & $2.51 \mathrm{e}^{-09}$ \\
\hline neuron projection & GO:0043005 & 651 & 17 & $1.36 \mathrm{e}^{-08}$ \\
\hline cytoplasmic vesicle & GO:0031410 & 928 & 19 & $5.32 \mathrm{e}^{-08}$ \\
\hline vesicle & GO:0031982 & 970 & 19 & $1.12 \mathrm{e}^{-07}$ \\
\hline cytoplasmic membrane-bounded & GO:0016023 & 862 & 18 & $1.27 \mathrm{e}^{-07}$ \\
\hline vesicle & & & & \\
\hline cytoskeleton & GO:0005856 & 1790 & 25 & $1.28 \mathrm{e}^{-07}$ \\
\hline membrane-bounded vesicle & GO:0031988 & 883 & 18 & $1.87 \mathrm{e}^{-07}$ \\
\hline
\end{tabular}


6

LRRK2 interactors enrichment for "GO cell component" and "GO biological process" terms.

The LRRK2 filtered interactome is shown after WebGestalt enrichment analysis. The enriched GO terms were grouped in: transport/localization, cell organization, regulation of kinase activity, cytosol, vesicles, cytoskeleton and cell projections. Every protein in the filtered interactome was connected to the $\mathrm{GO}$ term groups it has participated toward the enrichment. In the table the 7 groups are listed in columns (A-G) and rows (1-7). The percentage of proteins from the LRRK2 interactome that contributed toward the enrichment of the GO term group listed in the row and that were also reported in the GO term group reported in the column was calculated (intersection between the group in the row and the group in the column). In the last row of the table the percentage of the proteins that contributed toward the enrichment of each GO term group was calculated against the total number of LRRK2 interactors (i.e. 62 proteins). Cells discussed in the text are highlighted. 


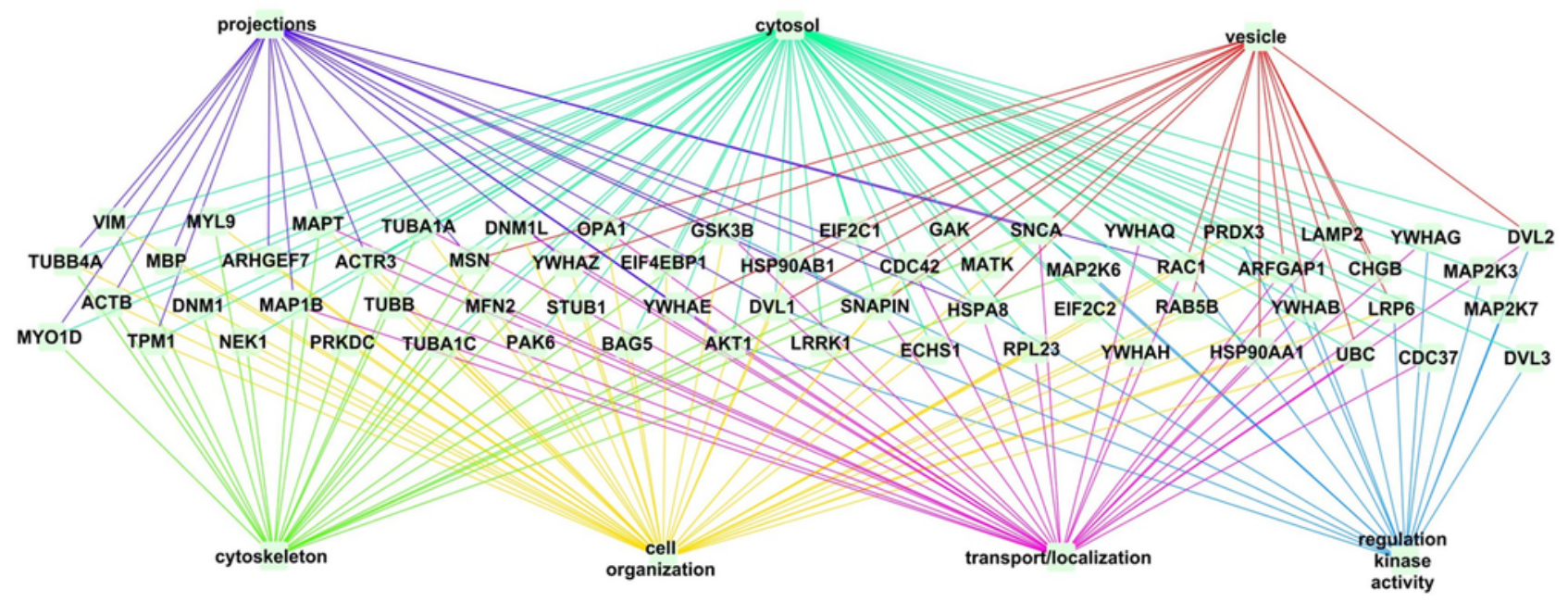

\begin{tabular}{|c|c|c|c|c|c|c|c|}
\hline & 1 & 2 & 3 & 4 & 5 & 6 & 7 \\
\hline & vesicles & projections & cytoskeleton & cytosol & transport & cell organization & reg. kinase \\
\hline vesicles & $100 \%$ & $53 \%$ & $32 \%$ & $100 \%$ & $79 \%$ & $74 \%$ & $47 \%$ \\
\hline projections & $43 \%$ & $100 \%$ & $61 \%$ & $96 \%$ & $61 \%$ & $87 \%$ & $26 \%$ \\
\hline cytoskeleton & $24 \%$ & $56 \%$ & $100 \%$ & $100 \%$ & $60 \%$ & $84 \%$ & $24 \%$ \\
\hline cytosol & $32 \%$ & $37 \%$ & $42 \%$ & $100 \%$ & $53 \%$ & $68 \%$ & $31 \%$ \\
\hline transport & $48 \%$ & $45 \%$ & $48 \%$ & $100 \%$ & $100 \%$ & $81 \%$ & $42 \%$ \\
\hline cell organization & $33 \%$ & $48 \%$ & $50 \%$ & $95 \%$ & $60 \%$ & $100 \%$ & $26 \%$ \\
\hline reg. kinase & $50 \%$ & $33 \%$ & $33 \%$ & $100 \%$ & $72 \%$ & $61 \%$ & $100 \%$ \\
\hline total in & $31 \%$ & $37 \%$ & $40 \%$ & $95 \%$ & $50 \%$ & $68 \%$ & $29 \%$ \\
\hline LRRK2 interactome & & & & & & & \\
\hline
\end{tabular}




\section{7}

"GO biological process" terms enriched for LRRK2 interactors in GO supported by Panther.

Pie charts showing the composition of every significance level of enrichment. The legend shows the $12 \mathrm{GO}$ term groups, although in the charts the group name was reported only for those that reached the $10 \%$ contribution toward the enrichment.

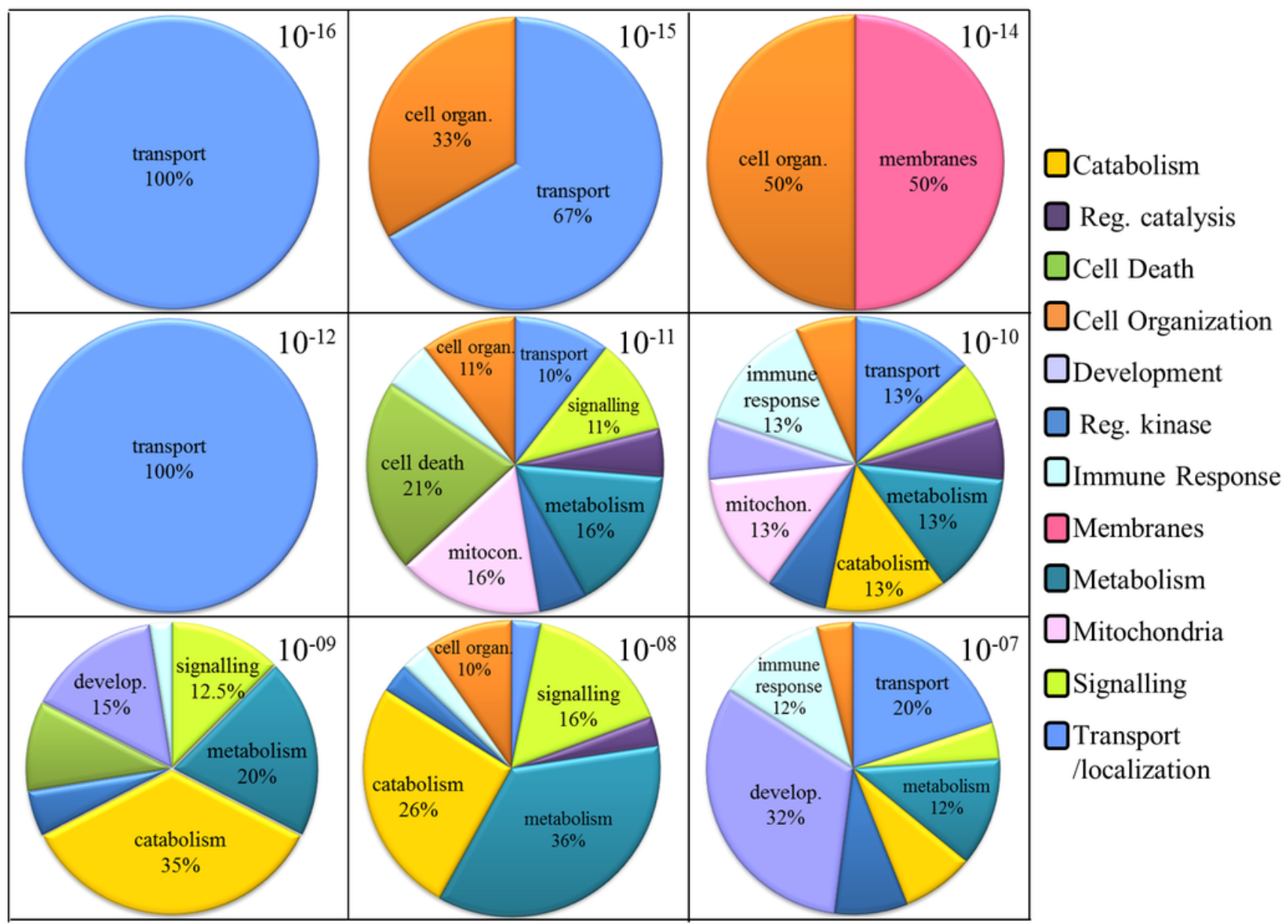




\section{8}

Annotations for LRRK2 self-interaction.

LRRK2 in the middle of the graph is linked to the first author of publications describing selfinteraction. Different colours represent different methods used to infer the interaction; note that since IntAct and BioGRID use different classifications for the interaction detection method, a simplified and harmonized version has been applied to this figure to help the reader. In particular: i) Affinity Capture stands for - Affinity Capture-Western (in BioGRID) Anti Tag Coimmunoprecipitation, and Pull Down (in IntAct); ii) Biochemical Activity stands for

- Biochemical Activity (in BioGRID) - Protein Kinase Assay (in IntAct).

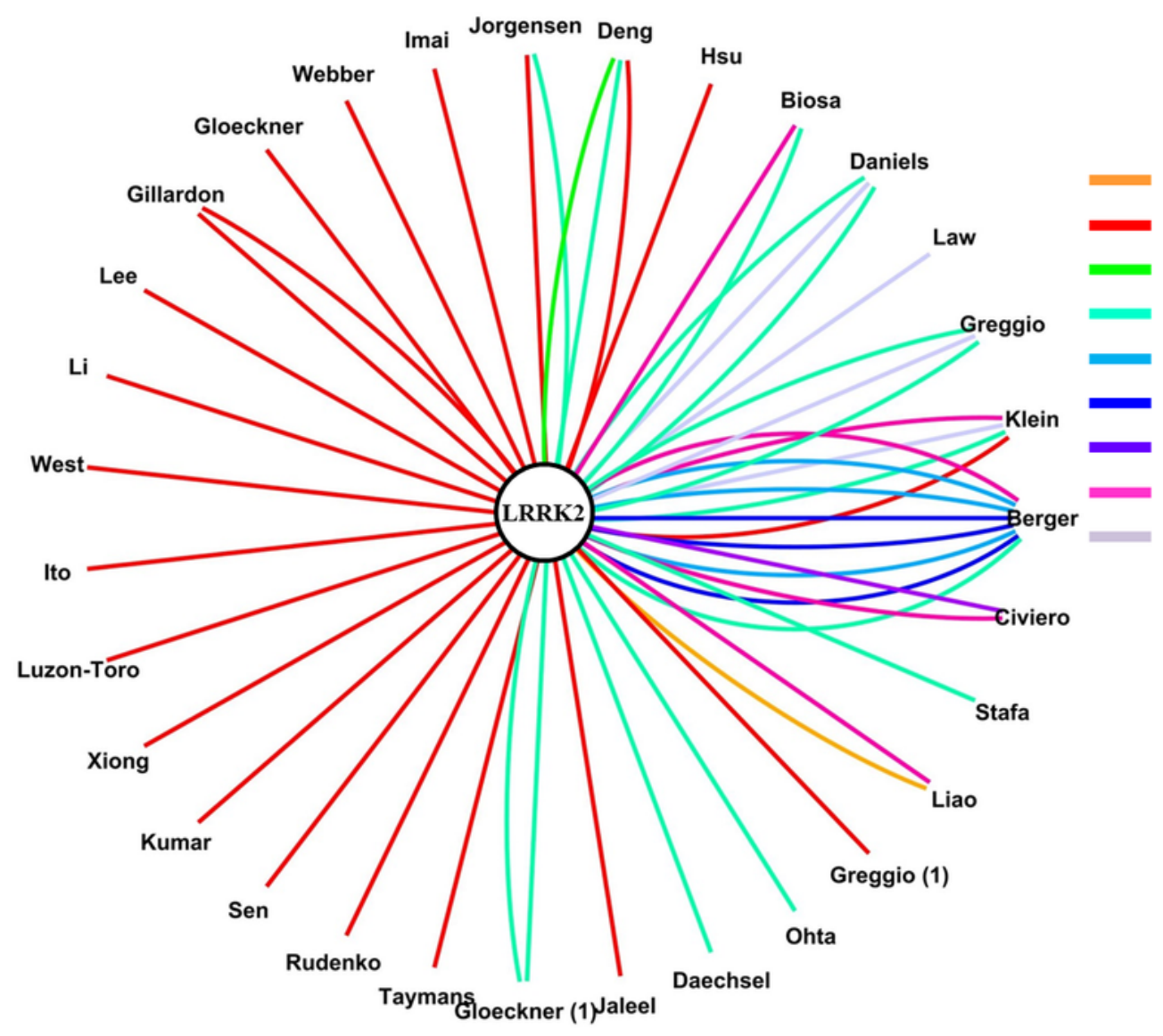

Light Scattering

Biochemical Activity Co-crystal Structure Affinity Capture Co-sedimentation Cross-linking Electron Microscopy Molecular Sieving Two-hybrid 


\section{9}

Profile of LRRK2 self-interaction.

Each residue described as involved in LRRK2 self-interaction is reported with a dot, the dimension of the dot is proportional to the number of publications annotating that specific residue. A profile covering the entire length of the LRRK2 protein is shown in blue, in the bottom half of the image; the $y$ value of the profile represents the number of publications in which the fragment in the profile was reported as associated with LRRK2 self-interaction.

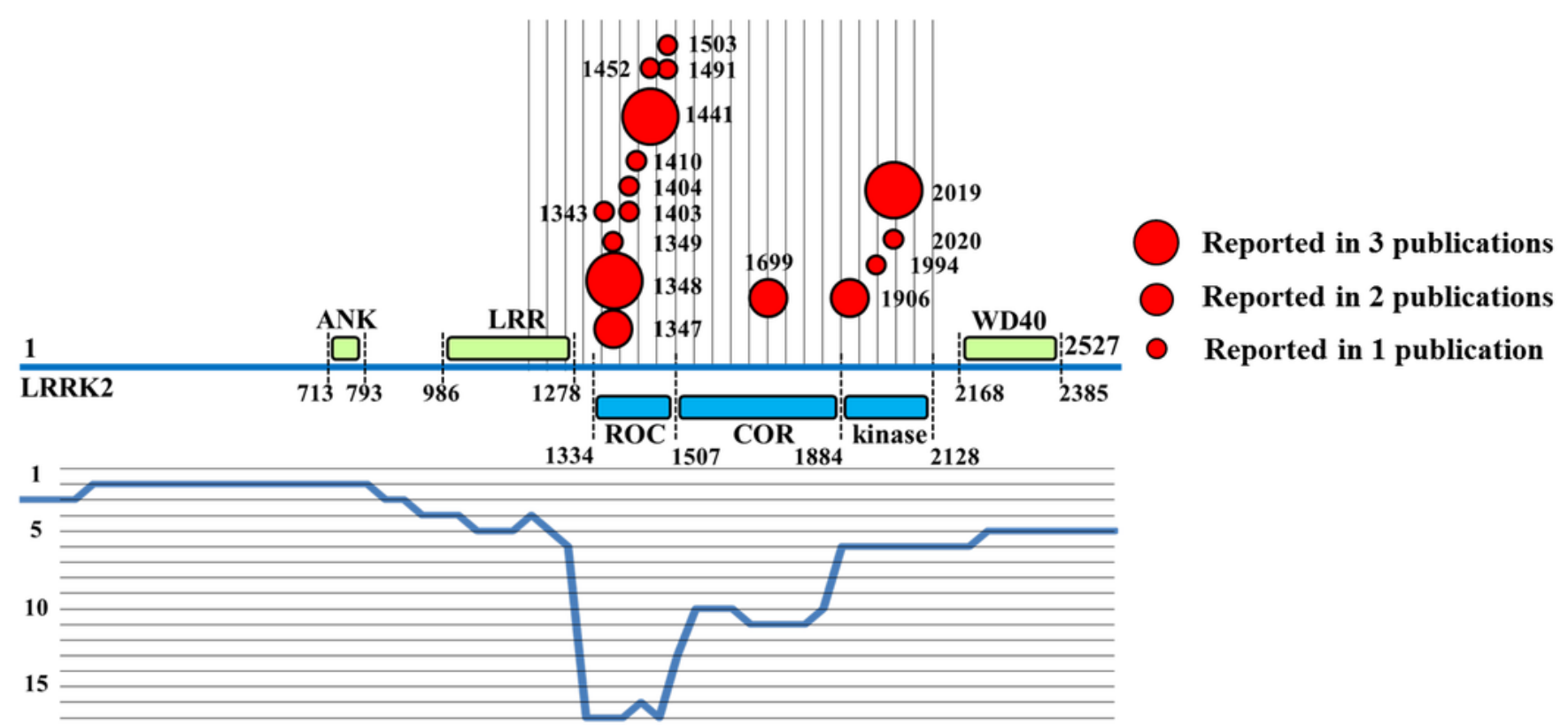


Table $\mathbf{1}$ (on next page)

LRRK2 interactors associated to the genetics of PD or IBD.

A, number of annotations; P, number of publications; HTP, one of the detection methods is high throughput. The approximate distance in base pair between the risk SNP and the protein coding sequence was calculated and the protein is indicated as candidate protein according to the original GWAs study. Methods are as follows: IP, immunoprecipitation; CM, confocal microscopy; FM, fluorescent microscopy; PA, protein array; BA, biochemical activity; MS, affinity capture mass spectrometry and $2 \mathrm{H}$, two hybrid. 


\section{Table 1}

3

\begin{tabular}{|c|c|c|c|c|c|c|c|c|c|c|}
\hline Protein & A & $\mathrm{P}$ & Methods & HTP & $\begin{array}{c}\text { Familial } \\
\text { PD }\end{array}$ & $\begin{array}{c}\text { GWA } \\
\text { PD }\end{array}$ & $\begin{array}{l}\text { GWA } \\
\text { IBD }\end{array}$ & $\begin{array}{l}\text { Associated } \\
\text { SNP }\end{array}$ & $\begin{array}{c}\text { Distance } \\
(\mathrm{Kbp})\end{array}$ & Candidate \\
\hline SNCA & 6 & 2 & IP, CM & - & $\mathbf{X}$ & $\mathbf{X}$ & & rs356182 & $\sim 20$ & Yes \\
\hline RAB7L1 & 5 & 1 & $\begin{array}{l}\text { PA, IP, } \\
\text { FM, CM }\end{array}$ & + & & $\mathbf{X}$ & & rs823118 & $\sim 14$ & Yes \\
\hline GAK & 5 & 2 & $\begin{array}{l}\text { PA, IP, } \\
\text { FM, CM }\end{array}$ & + & & $\mathbf{X}$ & & rs34311866 & $\sim 25$ & Yes \\
\hline MAPT & 7 & 2 & IP, BA & - & & $\mathbf{X}$ & & rs17649553 & 0 & Yes \\
\hline CDC37 & 5 & 4 & MS, IP & + & & & $\mathbf{X}$ & rs11879191 & 0 & No \\
\hline DVL1 & 4 & 1 & $\begin{array}{c}\mathrm{IP}, \mathrm{CM} \\
2 \mathrm{H}\end{array}$ & + & & & $\mathbf{X}$ & rs 12103 & $\sim 23$ & No \\
\hline GNA12 & 1 & 1 & MS & + & & & $\mathbf{X}$ & rs798502 & 0 & Yes \\
\hline CALM3 & 1 & 1 & MS & + & & & $\mathbf{X}$ & rs1126510 & $\sim 9.7$ & Yes \\
\hline GLTPD1 & 1 & 1 & PA & + & & & $\mathbf{X}$ & rs 12103 & $\sim 13$ & No \\
\hline CEP72 & 1 & 1 & PA & + & & & $\mathbf{X}$ & rs11739663 & $\sim 18$ & No \\
\hline ARPC2 & 1 & 1 & MS & + & & & $\mathbf{X}$ & rs2382817 & $\sim 32$ & Yes \\
\hline RIPK2 & 1 & 1 & BA & - & & & $\mathbf{X}$ & rs7015630 & $\sim 73$ & Yes \\
\hline PSMG1 & 1 & 1 & PA & + & & & $\mathbf{X}$ & rs 2836878 & $\sim 82$ & No \\
\hline DBN1 & 1 & 1 & MS & + & & & $\mathbf{X}$ & rs 12654812 & $\sim 89$ & No \\
\hline RPAP3 & 1 & 1 & PA & + & & & $\mathbf{X}$ & rs11168249 & $\sim 108$ & No \\
\hline CD2BP2 & 1 & 1 & PA & + & & & $\mathbf{X}$ & rs11150589 & $\sim 116$ & No \\
\hline PLK1 & 1 & 1 & PA & + & & & $\mathbf{X}$ & rs 7404095 & $\sim 163$ & No \\
\hline ACTR2 & 1 & 1 & MS & + & & & $\mathbf{X}$ & rs6740462 & $\sim 169$ & No \\
\hline STIP1 & 1 & 1 & PA & + & & & $\mathbf{X}$ & rs559928 & $\sim 178$ & No \\
\hline MYO1B & 1 & 1 & MS & + & & & $\mathbf{X}$ & rs 1517352 & $\sim 179$ & No \\
\hline CLTC & 1 & 1 & MS & + & & & $\mathbf{X}$ & rs 1292053 & $\sim 189$ & No \\
\hline
\end{tabular}

